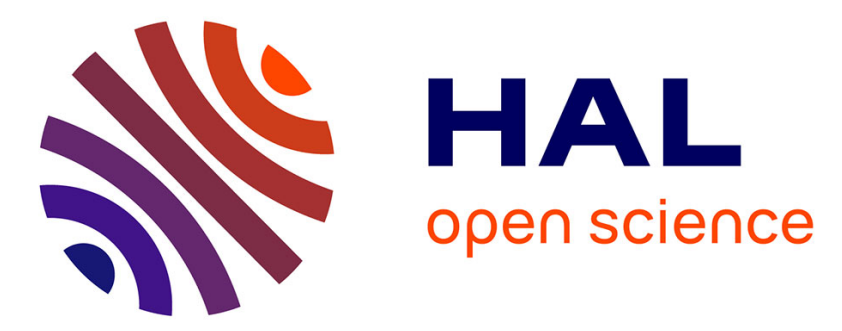

\title{
A new method for the detection of using flow cytometry
}

J. Barbosa, C. Bragada, S. Costa-De-Oliveira, E. Ricardo, A. G. Rodrigues, C. Pina-Vaz

\section{To cite this version:}

J. Barbosa, C. Bragada, S. Costa-De-Oliveira, E. Ricardo, A. G. Rodrigues, et al.. A new method for the detection of using flow cytometry. European Journal of Clinical Microbiology and Infectious Diseases, 2010, 29 (9), pp.1147-1152. 10.1007/s10096-010-0980-5 . hal-00600769

\section{HAL Id: hal-00600769 \\ https://hal.science/hal-00600769}

Submitted on 16 Jun 2011

HAL is a multi-disciplinary open access archive for the deposit and dissemination of scientific research documents, whether they are published or not. The documents may come from teaching and research institutions in France or abroad, or from public or private research centers.
L'archive ouverte pluridisciplinaire HAL, est destinée au dépôt et à la diffusion de documents scientifiques de niveau recherche, publiés ou non, émanant des établissements d'enseignement et de recherche français ou étrangers, des laboratoires publics ou privés. 
Diseases

Editorial Manager(tm) for European Journal of Clinical Microbiology \& Infectious

Manuscript Draft

Manuscript Number: EJCMID-D-09-00424R2

Title: A new method for detection of Pneumocystis jirovecii using flow cytometry

Article Type: Article

Keywords: Pneumocystis jirovecii 1; Flow cytometry 2; Epifluorescence microscopy 3; Monoclonal antibodies 4; Pneumonia by Pneumocystis 5

Corresponding Author: Dr Joana Barbosa,

Corresponding Author's Institution:

First Author: Joana Barbosa

Order of Authors: Joana Barbosa; Cláudia Bragada; Sofia Costa-de-Oliveira; Elisabete Ricardo; Acacio Gonçalves Rodrigues; Cidalia Pina-Vaz

Abstract: Pneumocystis jirovecii is an opportunistic pathogen responsible for severe pneumonia in immunocompromised patients. Its diagnosis has been based upon direct microscopy either by classic staining (Gomori Grocott) or by epifluorescence microscopy (IFS), both time-consuming and low on sensitivity. Our aim was to develop a Flow Cytometric (FC) protocol for detection of P. jirovecii on respiratory samples. In our study, 420 respiratory samples were analysed in parallel by IFS and FC, and compared from clinical diagnosis to its resolution upon specific anti-Pneumocystis therapy. The optimum specific antibody concentration for FC analysis was determined as $10 \mu \mathrm{g} / \mathrm{ml}$, without any cross-reactions to bacteria or fungi. All positive cases detected by IFS were positive by FC, however FC considered 8 samples positive which were classified as negative by routine technique. These samples arose from patients with respiratory symptoms who responded favourably to Pneumocystis specific therapy and were subsequently considered true positive. Using clinical diagnosis as a reference method, FC showed $100 \%$ of sensitivity and specificity, whereas IFS showed $90.9 \%$ of sensivity and $100 \%$ of specificity. According to our results, a new diagnostic approach is now available to detect $P$. jirovecii in respiratory samples.

Response to Reviewers: Dear Doctor Alex van Belkum Editor-in-Chief of European Journal of Clinical Microbiology \& Infectious Diseases

Thank you for your comments and for considering our manuscript EJCMID-D-09-00424R1 for publication. We have attended to all comments and made the recommended changes. About the major comment, for the 420 samples, 130 were from HIV positive patients being $35(27 \%)$ positive by both methods. The percentage of positive samples on non HIV population was $18 \%$ (53). So, we included these data on Material and Methods (line 5 - page 5) and also compared the positive percentage found on HIV and non HIV population in the Results (line 24, 25 - page 7). We also introduce a new reference (D'Avignon LC, Schofield CM, Hospenthal DR (2008) Pneumocystis Pneumonia. Semin Respir Crit Care Med 29:132-140) to validate the results found from positive samples of HIV patients. The manuscript was corrected by a native English speaker. We hope that the paper could now be considered acceptable for publication. 
Regarding specific comments

-Abstract: We have accepted all your suggestions and made all the necessary changes

-Introduction: We have replaced and shortened the sentence according your suggestion

-Discussion: We have changed "visual" by "microscopic".

Best regards

Joana Barbosa 
Dear Doctor Alex van Belkum

Editor-in-Chief of European Journal of Clinical Microbiology \& Infectious Diseases

Thank you for your comments and for considering our manuscript EJCMID-D-09-00424R1 for

publication. We have attended to all comments and made the recommended changes. About the major comment, for the 420 samples, 130 were from HIV positive patients being $35(27 \%)$ positive by both methods. The percentage of positive samples on non HIV population was $18 \%$ (53). So, we included these data on Material and Methods (line 5 - page 5) and also compared the positive percentage found on HIV and non HIV population in the Results (line 24, 25 - page 7). We also introduce a new reference (D'Avignon LC, Schofield CM, Hospenthal DR (2008) Pneumocystis Pneumonia. Semin Respir Crit Care Med 29:132-140) to validate the results found from positive samples of HIV patients. The manuscript was corrected by a native English speaker. We hope that the paper could now be considered acceptable for publication.

\section{Regarding specific comments}

- Abstract: We have accepted all your suggestions and made all the necessary changes

- Introduction: We have replaced and shortened the sentence according your suggestion

- Discussion: We have changed "visual" by "microscopic".

Best regards 


\title{
(*) CORRESPONDENT FOOTNOTE
}

Joana Margarida Moutinho Barbosa

Department of Microbiology, Porto Faculty of Medicine, University of Porto

\begin{abstract}
Al. Prof. Hernâni Monteiro
\end{abstract}
4200 - 319 Porto PORTUGAL

Phone: +351225513662

Fax: +351225513662

Email: gui75@sapo.pt 


\section{AbSTRACT}

1 3 4 5 6 7 8 9 106 11

Pneumocystis jirovecii is an opportunistic pathogen responsible for severe pneumonia in immunocompromised patients. Its diagnosis has been based upon direct microscopy either by classic staining (Gomori Grocott) or by epifluorescence microscopy (IFS), both time-consuming and low on sensitivity. Our aim was to develop a Flow Cytometric (FC) protocol for detection of P. jirovecii on respiratory samples. In our study, 420 respiratory samples were analysed in parallel by IFS and FC, and compared from clinical diagnosis to its resolution upon specific anti-Pneumocystis therapy. The optimum specific antibody concentration for FC analysis was determined as $10 \mu \mathrm{g} / \mathrm{ml}$, without any cross-reactions to bacteria or fungi. All positive cases detected by IFS were positive by FC, however FC considered 8 samples positive which were classified as negative by routine technique. These samples arose from patients with respiratory symptoms who responded favourably to Pneumocystis specific therapy and were subsequently considered true positive. Using clinical diagnosis as a reference method, FC showed $100 \%$ of sensitivity and specificity, whereas IFS showed $90.9 \%$ of sensivity and $100 \%$ of specificity. According to our results, a new diagnostic approach is now available to detect $P$. jirovecii in respiratory samples.

Keywords: Pneumocystis jirovecii; flow cytometry; epifluorescence microscopy; monoclonal antibodies; pneumonia by Pneumocystis 


\section{INTRODUCTION}

12

3 $4^{3}$

5

64 7

Pneumocystis jirovecii (previously named Pneumocystis carinii) is an atypical fungus. For 80 years it was considered a protozoan, since its diagnostic form is a cyst, but ribosomal RNA and DNA studies demonstrated similarities to fungus [1]. In 2001 this microorganism was officially reclassified as a fungus belonging to the class Archiascomycetes [1], and renamed P. jirovecii [2]. In humans, this pathogen is responsible for an opportunistic infection at the lower respiratory tract, but it can lead to severe Pneumocystis pneumonia (PCP) in immunocompromised patients, particularly in those with AIDS [3-4]. Prior to the AIDS epidemic, $P$. jirovecii had been sporadically reported as a cause of death in malnourished infants and responsible for epidemics in institutions like nursing homes and hospitals [5]. Currently, it represents the most frequent AIDS opportunistic pathogen, although PcP incidence has decreased significantly due to the extensive use of highly active antiretroviral therapy (HAART) and prophylactic treatment [4-6]. PCP may also represent a small fraction of respiratory infections in patients with pulmonary diseases such as chronic obstructive pulmonary disease (DPCO) or displaying immunity conditions like malignancy [7-8].

Over the past fifteen years, $P$. jirovecii carriage in healthy subjects has been widely demonstrated through the presence of anti - P. jirovecii antibodies in their serum, with serologic analysis being used simply for assessment of the infection epidemiology [9]. PCP however is considered to result from de novo infection rather than from reactivation of a latent infection [6]. It is assumed that human transmission of $P$. jirovecii occurs through airborne route, as suggested by studies performed in rodent models [6]. Nonetheless, interindividual transmission has been suggested due to the occurrence of PCP case clusters in hospitals, namely in paediatrics, hematology - oncology, intensive care, transplantation unit and infectious diseases units [10]. P. jirovecii may be exhaled to the environment from infected patients, as demonstrated from cyst DNA detection in air filters from hospital rooms of patients who developed PCP [10].

The detection of $P$. jirovecii in an infected patient is based upon the direct visualization of organisms (cysts, sporocysts or trophozoites) in clinical specimens, such as bronchoalveolar lavage (BAL), induced sputum (IS) or lung biopsy, which is the gold standard specimen $[6,11]$. Although specific fluorescent staining techniques were developed for assessment under fluorescent microscopy, such methods are very time-consuming, too cumbersome and subject to human error, especially when samples yield a low number of $P$. jirovecii organisms [11]. Molecular studies such as polymerase chain reaction (PCR) presented a higher sensitivity and 
1 variable specificity when compared to microscopic detection of P. jirovecii in BAL [12-13]. However, PCR

${ }_{2}^{1} 2$ remains unavailable in most clinical microbiology laboratories and is an expensive technique [14-15]. More

${ }_{4}^{3}$ so, a variable specificity has been described ranging from 62.5 to $100 \%$ depending on the type of specimen 64 [14-15]. Distinct applications of Flow Cytometry (FC) in Microbiology have been developed by our group in 7

85 order to increase the diagnostic sensitivity in clinical samples [16-18]. In the present study we have developed 9 10 11 
MATERIAL AND METHODS

12

3 $4^{3}$ 5 64 7

\section{Clinical SPECIMEN}

A total of 420 samples, including 380 bronchoalveolar lavage (BAL) and bronchial washing (BW) specimens, and 40 bronchial secretion (BS) aspirates were prospectively collected from patients with different immunological conditions. From those 420 patients, 130 were HIV positive. Samples were kindly provided by the Microbiology Laboratories of several Portuguese hospitals namely Hospital São João (HSJ, Porto, Portugal), Instituto Português de Oncologia Francisco Gentil (IPOFG, Porto, Portugal), Hospital Joaquim Urbano (HJU, Porto, Portugal) and Centro Hospitalar de Coimbra (CHC, Coimbra, Portugal). A maximum of $30 \mathrm{~mL}$ of $\mathrm{BAL}$ or $\mathrm{BW}$, and 2 to $4 \mathrm{~mL}$ of $\mathrm{BS}$ samples were placed in a sterile container, and frozen at $-70^{\circ} \mathrm{C}$ for further processing for immunofluorescence staining (IFS) and flow cytometry (FC) analysis.

\section{SAMPLE PREPARATION}

For IFS, clinical samples were mixed with mucolityc agent $\mathrm{N}$ - acetyl - L - cystein (Merck ${ }^{\text {) }}$ and incubated in a water bath at $37^{\circ} \mathrm{C}$ for 20 minutes or until complete dissolution, followed by centrifugation at $3000 \mathrm{~g}$ for 15 min. Subsequently, samples were washed twice with sterile water $\left(\mathrm{H}_{2} \mathrm{O}\right)$, centrifuged as above and the sediment ressuspended in a small volume of water which was divided into two portions; one portion was placed on a glass slide, air dried and fixed while the other portion was used for FC analysis.

\section{IMMUNOFLUORESCENCE STAINING}

Smears were stained according to the manufacturer's instructions with the Detect $\mathrm{IF}^{\mathrm{TM}}$ kit Pneumocystis carinii (Axis - Shield Diagnostics Limited, United Kingdom) and analysed under an epifluorescence microscope Leitz Laborlux K (Leica, NY, U.S.A.). Smears were considered positive for P. jirovecii whenever two or more green cysts were visualized, whether isolated or in a group.

\section{OPTIMIZATION OF A Flow CyTOMETRY PROTOCOL}

For optimization of the FC protocol, a mix of four positive samples and a mix of four negative samples (evaluated by IFS) were prepared. Samples were divided into two equal aliquots and one of them was passed through a filter with $30 \mu \mathrm{m}$ pore size (Partec CellTrics $\left.{ }^{\circledR}\right)$. Twenty microliters of specific enzyme from Detect $\mathrm{IF}^{\mathrm{TM}}$ kit Pneumocystis carinii (Axis - Shield Diagnostics Limited, United Kingdom) were added to $100 \mu$ l of the respiratory sample and incubated at $37^{\circ} \mathrm{C}$ for 30 minutes; $500 \mu \mathrm{l}$ of sterile $\mathrm{H}_{2} \mathrm{O}$ were then added and a subsequent centrifugation was performed at $3000 \mathrm{~g}$ for 5 minutes. 
1 One hundred microliters of centrifuged sample were stained with serial concentrations $(0,5.0,10.0,15.0$ and

$20.0 \mu \mathrm{l}$ ) of specific P. jirovecii mouse monoclonal antibody (Axis - Shield Diagnostics Limited, United Kingdom), followed by dark incubation at $37^{\circ} \mathrm{C}$ for 15 minutes; Subsequently, $500 \mu 1$ of sterile $\mathrm{H}_{2} \mathrm{O}$ were added and centrifugation at $3000 \mathrm{~g}$ for 5 min was performed; the supernantant was discarded and the pellet ressuspended in $100 \mu$ of sterile $\mathrm{H}_{2} \mathrm{O}$, vortexed for 30 s, transferred to a propylene tube and analyzed by FC.

\section{FLOW CYTOMETRY ANALYSIS}

The optical characteristics of the cyst suspensions were evaluated on a FACSCalibur Cytometer (BD Biosciences, Sydney) standard model equipped with 3 photomultiplayers (PMTs), standard filters (FL1: BP 530/30 nm; FL2: BP 585/42 nm; FL3: LP $650 \mathrm{~nm}$ ), a $15 \mathrm{~mW} 488 \mathrm{~nm}$ Argon Laser and with cell Quest Pro software (version 4.0.2, BD Biosciences, Sydney). Operating conditions included log scales on all detectors (forward scatter (FSC), side scatter (SSC), and fluorescence detectors (FL1)). Acquisition settings were defined using a non-stained sample (autofluorescence) and adjusting the PMTs voltage to the first logarithmic (log) decade. Instrument controls followed the described standard procedures by the manufacturer.

\section{ASSESSMENT OF SENSITIVITY AND SPECIFICITY OF FC STAINING PROTOCOL}

To assess specificity, positive and negative IFS samples for $P$. jirovecii were mixed with $1 \times 10^{6}$ cells $/ \mathrm{ml}$ of different microorganisms, namely Escherichia coli type strain ATCC 35218 from the American Type Culture Collection (ATCC), Staphylococcus aureus ATCC 25923 and Candida albicans ATCC 10231. The mix was stained with the specific $P$. jirovecii antibody under optimized conditions and analysed by FC.

\section{EVALUATION OF Clinical RESPIRATORY SAMPLES}

All clinical samples were analysed both by IFS and by FC according to the previously optimized conditions and the results were subsequently compared.

\section{DATA COMPARISON}

Results of IFS were compared to those obtained by FC. Clinical diagnosis was used as the standard. When results were discrepant, clinical symptoms and signs were considered; patients with oxygen level $\mathrm{PaO}_{2}<70$ $\mathrm{mmHg}$, an X-ray depicting a diffuse bilateral infiltrate and with a favourable outcome upon specific therapy were considered as true positive cases for PCP. 
RESUlts

Respiratory samples were considered positive for P. jirovecii when two or more green round formations, namely cysts, were observed on epifluorescence microscopy. By IFS, 340 samples were negative and 80 samples were positive for $P$. jirovecii.

Since there is not an available commercial type strain of $P$. jirovecii, we used a mix of four IFS positive and four IFS negative samples (without cysts) as material for optimization of the Flow Cytometric (FC) protocol. As we increased the specific antibody concentrations, an increase of the Mean Intensity of Fluorescence (MIF) was also clear. Twenty $\mu 1$ of specific-antibody resulted in the highest value of MIF at FL1 although a similar staining pattern was obtained with 10.0 or $15.0 \mu \mathrm{l}$ (Figure 1). As such, all further experiments were carried out with the lowest concentration sufficient to detect the organisms from the autofluorescence background i.e. 10.0 $\mu 1$.

To determine the specificity of the staining, microorganisms usually present in respiratory samples were mixed with negative and positive control samples, which were then incubated with the monoclonal antibody. Although an increase in events was detectable in the presence of high concentrations of bacteria, those events were not selected in our settings due to their smaller size; yeasts appeared near the acquisition gate corresponding to $P$. jirovecii cysts but displayed much lower fluorescence intensity than that of the stained cysts (Figure 2).

Upon optimization of the cytometric protocol, clinical samples were analysed. Sample preparation included mucolytic agent, with and without filtration (30 $\mu \mathrm{m}$ pore size). A greater number of non-fluorescent cytometric events occurred on samples without filtration leading to difficulties in its interpretation. A better separation of stained cysts from debris was obtained upon filtration without signal lost (Figure 3). For FC analysis, we considered positive all the respiratory samples whose events appeared on the acquisition gate $\mathrm{R}$ corresponding to P. jirovecii cysts stained with specific antibody (typical example shown on Figure 3).

Through FC we found 88 positive samples, 35 of which belonged from HIV patients (27\%). Eighty positive cases (19\%) were detected by both IFS and FC methods. However, 8 clinical samples previously considered negative by IFS, were found to be positive by FC assay (Table 1). All 8 cases were from HIV positive patients with clinical diagnostic criteria of PCP such as fever, cough, shortness of breath, weight loss associated with pulmonary infiltrates on chest x-ray, and an arterial oxygen low level. In addition, the patients had improved 
1 upon specific treatment with trimethoprim despite negative IFS. Considering the clinical criteria as the ${ }_{2}^{1} 2$ diagnostic standard, such cases should be considered as true positive. According to our study FC analysis ${ }_{4}^{3} 3$ displays $100 \%$ sensitivity and specificity versus $90.9 \%$ sensitivity and $100 \%$ specificity of IFS assay. 64 Consequently, IFS presented a predictive positive value of $100 \%$ and a predictive negative value of $97.6 \%$. 7 85 9 10 


\section{DISCUSSION}

Specific and prompt diagnosis of Pneumocystis jirovecii infection has significant clinical relevance since this opportunistic organism can cause severe pneumonia in immunocompromised patients [6]. As this organism cannot be cultured in vitro, the standard diagnostic procedure for detection of $P$. jirovecii relies upon the direct microscopic examination of the clinical specimen. Using commercialized fluorescein or enzyme-labelled monoclonal or polyclonal Pneumocystis antibodies in respiratory samples, a higher detection sensitivity can be achieved [11]. Considering the increasing complexity of diagnostic laboratory techniques, resulting in higher costs and the need of human expertise, the development of more sensitive and automated methods which allow a rapid assessment of clinical specimens is highly recommended. Flow cytometry (FC) is a method based upon the evaluation of cell fluorescence, frequently shown to be specific and yielding great sensitivity. Moreover, it allows the detection of low threshold limits, difficult to obtain by standard methods [16-19]. The first cytometric study regarding the formally $P$. carinii was developed by Libertin and colleagues [19] in lung homogenates samples from infected mice and humans, however without practical application. Later assays were performed by Lapinsky and colleagues [20] in order to observe the pharmacological action of drugs upon growth and viability of these microorganisms in animal models. The flow cytometer is an expensive equipment present in most immunology laboratories that could probably be easily shared by microbiologists. Since the amount of specific monoclonal antibody is lower than that used for IFS, it has the potential to be more economic.

Our study aimed to develop and optimize the cytometric analytical protocol regarding clinical samples. An important condition should be fulfilled: the concentration of the probe should be at its minimum to allow a clear separation of the stained population from all other events; while insufficient staining would not allow a clear separation from debris, excessive staining could result in unspecific fluorescence. Our study faced a number of limitations such as the absence of a commercially available type strain as well as the relative low number of positive samples, often containing very few organisms, which made it difficult to assess the sensitivity limit. Cytometric analysis could be difficult to perform in the presence of organic debris and as such, the filtration step prior to specific antibody staining resulted in an improved discrimination of debris from $P$. jirovecci organisms. In order to confirm the specificity of the monoclonal antibody used in this study, cross-reactions were investigated using both prokaryotic (E. coli, S. aureus) and eukaryotic microorganisms 
1 (C. albicans) mixed in a negative sample for P. jirovecii. An increased number of events was clear although ${ }_{2}^{1} 2$ without staining with the specific antibody (only fungi showed a very slightly increase in green fluorescence), demonstrating that the test is quite specific.

64 The results from the 420 clinical samples collected from putative PCP patients revealed that, in accordance to 7

85 literature reports, only $19 \%$ of the samples were positive by both methods (IFS and CF), possibly due to the 10 11

extensive use of prophylaxis on risk population [21, 22]. However, regarding the HIV positive population the percentage we found is which agree with literature [21]. Comparing the results from FC to those of IFS several cases would have to be considered as false positives. However, taking into account medical criteria as final diagnosis those should be considered as true positives.

Thus, a new cytometric detection method is now available for a more reliable detection of $P$. jirovecii organisms in respiratory clinical samples. 


\section{ACKNOWLEDGMENTS}

12 J. Barbosa was supported by a PhD grant (SFRH/BD/44346/2008) from Fundação para a Ciência e a 3
4 Tecnologia (FCT).

5

64

7

8

9

10

11

12

13

14

15

16

17

18

19

20

21

22

23

24

25

26

27

28

29

30

31

32

33

34

35

36

37

38

39

40

41

42

43

44

45

46

47

48

49

50

51

52

53

54

55

56

57

58

59

60

61

62

63 


\section{REFERENCES}

12

3

1. Murray PR, Rosenthal KS, Pfaller MA (2005) Fungal classification, structure, and replication. In: Elsevier Mosby (ed) Medical Microbiology, $5^{\text {th }}$ ed. Philadelphia, Pennsylvania, pp 67-73

2. Stringer JR, Beard CB, Miller RF, Wakefield AE (2002) A new name (Pneumocystis jiroveci) for Pneumocystis from humans. Emerg Infect Dis 8:891-896.

3. Kelley CF, Checkley W, Mannino DM, Franco-Paredes C, Del Rio C, Holguin F (2009). Trends in hospitalization for AIDS-associated Pneumocystis jirovecii pneumonia in the United States (1986 to 2005). Chest. 136:190-197.

4. Su YS, Lu JJ, Perng CL, Chang FY (2008). Pneumocystis jirovecii pneumonia in patients with and without human immunodeficiency virus infection. J Microbiol Immunol Infect. 4:478-482.

5. Wazir JF, Ansari NA (2004) Pneumocystis carinii infection. Update and review. Arch Pathol Lab Med 128:1023-1027.

6. Krajicek BJ, Thomas CF Jr, Limper AH (2009). Pneumocystis pneumonia: current concepts in pathogenesis, diagnosis, and treatment. Clin Chest Med. 30:265-278

7. Morris A, Sciurba FC, Norris KA (2008) Pneumocystis: a novel pathogen in a chronic obstructive pulmonary disease? COPD 5:43-51.

8. Maschmeyer G, Beinert T, Buchheidt D, Cornley OA, Einsele H, Heinz W, Heussel CP, Kahl C, Kiehl M, Lorenz J, Hof H, Mattiuzzi G (2009) Diagnosis and antimicrobial therapy of lung infiltrates in febrile neutropenic patients: guidelines of the infectious diseases working party of German Society of Haematology and Oncology. Eur J Cancer. 45: 2462-2472

9. Morris A, netravali M, Kilng HM, Shipley T, Ross T, Sciurba FC, Norris KA (2008) Relationship of pneumocystis antibody response to severity of chronic obstructive pulmonary disease. Clin Infect Dis 47:64-68

10. Nevez G, Chabé M, Rabodonirina M, Virmaux M, Dei-Cas E, Hause PM, Totet A (2008) Nosocomial Pneumocystis jiroveci infections. Parasite 15:359-365.

11. Procop GW, Haddad S, Quinn J, Wilson ML, Henshaw NG, Reller LB, Artymyshyn RL, Katanik MT, Weinstein MP (2004) Detection of Pneumocystis jiroveci in respiratory specimens by four staining methods. $\underline{\mathrm{J}}$ Clin Microbiol 42:3333-3335.

12. Durand-Joly I, Chabé M, Soula F, Delhaes L, Camus D, Dei-Cas E (2005) Molecular diagnosis of Pneumocystis pneumonia. FEMS Immunol Med Microbiol 45:405-410. 
13. Jiancheng W, Minjun H, Yi-jun A, Lan S, Zengzhu G, Jianrong S, Xixiong K (2009). Screening Pneumocystis carinii pneumonia in non-HIV-infected immunocompromised patients using polymerase chain reaction. Diagn Microbiol Infect Dis. 64:396-401.

14. Alvarez-Martinez MJ, Miró JM, Valls ME, Moreno A, Rivas PV, Solé M et al (2006) Sensitivity and specificity of nested and real-time PCR for the detection of Pneumocystis jiroveci in clinical specimens. Diagn Microbiol Inf Dis 56:153-160

15. Robberts FJL, Liebowitz LD, Chalkley LJ (2007) Polymerase chain reaction detection of Pneumocystis jiroveci: evaluation of 9 assays. Diagn Microbiol Inf Dis 58:385-392.

16. Barbosa JM, Costa-de-Oliveira S, Rodrigues AG, Hanscheid T, Shapiro H, Pina-Vaz C (2008a) A flow cytometric protocol for detection of Cryptosporidium spp. Cytometry A 73:44-47.

17. Barbosa J, Costa-de-Oliveira S, Rodrigues AG, Pina-Vaz C (2008b) Optimization of a flow cytometry protocol for detection and viability assessment of Giardia lamblia. Travel Med Infect Dis 6:234-239.

18. Barbosa J, Rodrigues AG, Pina-Vaz C (2009) A cytometric approach for detection of Encephalitozoon intestinalis: an emergent agent. Clin. Vaccine Immunol 16:1021-1024.

19. Libertin CR, Woloschak GE, Wilson WR, Smith TF (1984) Analysis of Pneumocystis carinii cysts with a fluorescence-activated cell sorter. J Clin Microbiol 20:877-880.

20. Lapinsky SE, Glencross D, Car NG, Kallenbach JM, Zwi S (1991) Quantification and assessment of viability of Pneumocystis carinii organisms by flow cytometry. J Clin Microbiol 29:911-915.

21. D'Avignon LC, Schofield CM, Hospenthal DR (2008) Pneumocystis Pneumonia. Semin Respir Crit Care Med 29:132-140.

22. Morris A, Wei K, Afshar K, Huang L. Epidemiology and Clinical Significance of Pneumocystis Colonization. JID 2008:197 - 205 

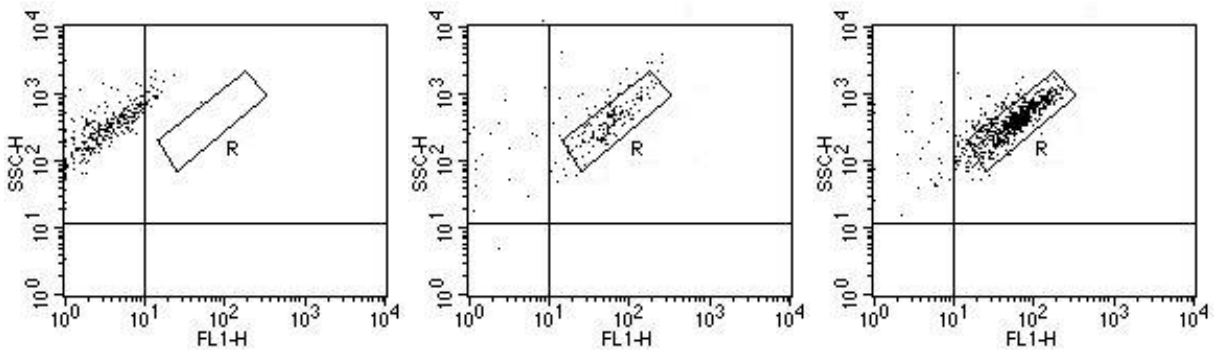

FIGURE 1 - Dot plot (side scatter (SSC) versus fluorescence intensity (FL1)) of a positive sample containing Pneumocystis jirovecii cysts labelled with serial concentrations of specific antibody Detect $\mathrm{IF}^{\mathrm{TM}} \mathrm{kit}^{\mathrm{m}}$ Pneumocystis carinii (Axis - Shield Diagnostics Limited, United Kingdom). a) without staining (autofluorescence); b) stained with $5.0 \mu \mathrm{g} / \mathrm{mL}$ of specific monoclonal antibody; c) stained with $10.0 \mu \mathrm{g} / \mathrm{mL}$ of specific monoclonal antibody. Region $\mathrm{R}$ corresponds to acquisition gate of $P$. jirovecii cysts stained with specific antibody.

8



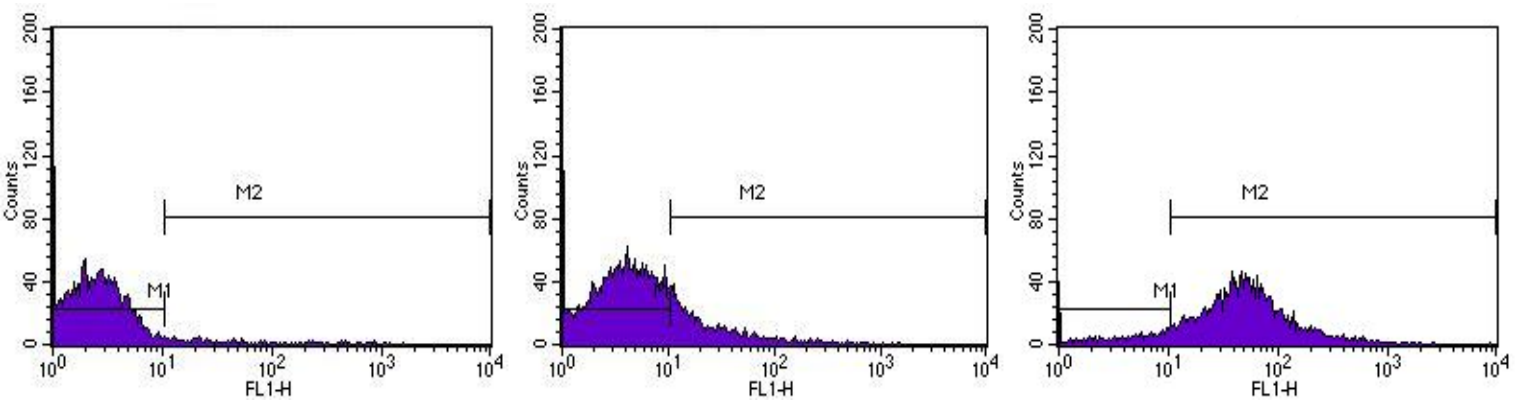

FIGURE 2 - Histogram correlating counts versus fluorescence intensity of Pneumocystis marker (FL1 - green fluorescence $530 \mathrm{~nm}$ ) of respiratory samples for Pneumocystis jirovecii: a) without staining (autofluorescence); b) negative sample stained with $10.0 \mu \mathrm{g} / \mathrm{mL}$ of specific monoclonal antibody and spiked with Candida albicans yeast; c) positive sample stained with $10.0 \mu \mathrm{g} / \mathrm{mL}$ of specific monoclonal antibody. Regions M1 and M2 correspond to autofluorescence and to specific antibody fluorescence, respectively. 


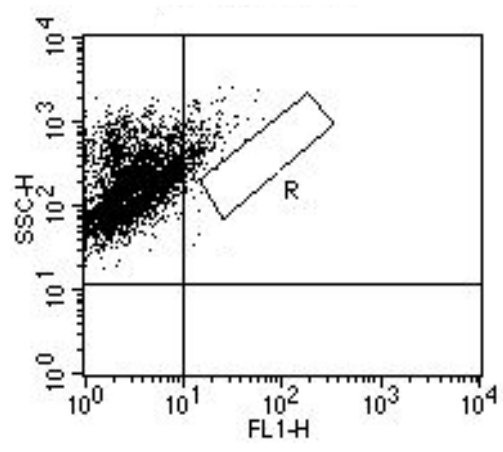

A

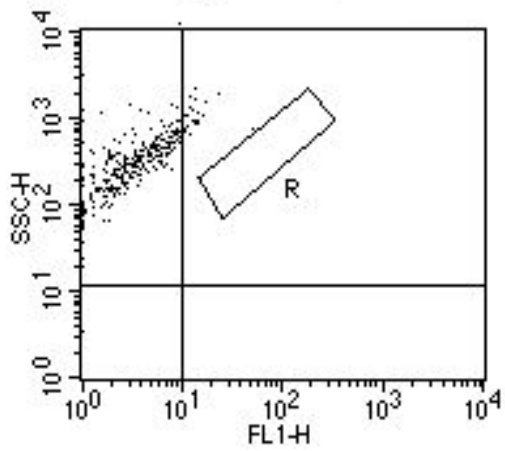

C

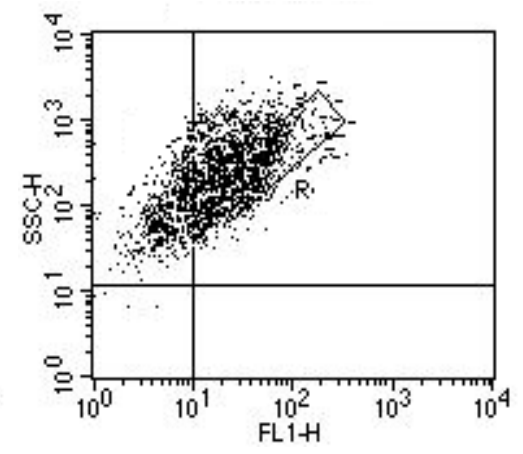

B

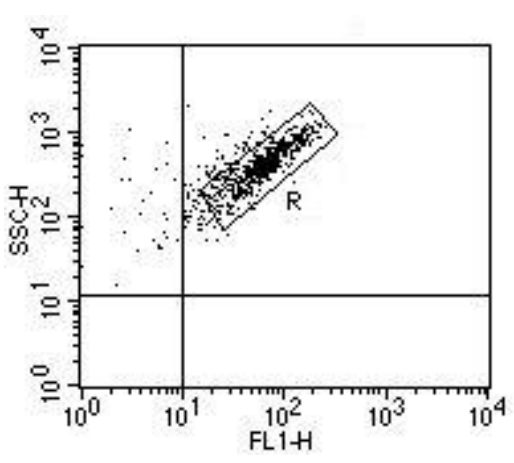

D

FIGURE 3 - Two-dimensional dot plot correlating a side scatter (SSC) versus fluorescence intensity of Pneumocystis marker (FL1 - green fluorescence $530 \mathrm{~nm}$ ) of respiratory samples for Pneumocystis jirovecii: A) negative sample without filtration; B) positive sample without filtration stained with $10.0 \mu \mathrm{g} / \mathrm{mL}$ of specific monoclonal antibody; C) negative sample upon filtration; D) positive sample upon filtration stained with 10.0 $\mu \mathrm{g} / \mathrm{mL}$ of specific monoclonal antibody. Region $\mathrm{R}$ corresponds to acquisition gate of $P$. jirovecii cysts stained with specific antibody. 
1 TABLE 1 - Results obtained from 420 respiratory samples for $P$. jirovecii diagnosis by Immunofluorescence ${ }_{2}^{1} 2$ (IFS) and Flow Cytometry (FC).

3

43

5

6

7

8

9

10

11

12

13

14

15

16

17

18

19

20

21

22

23

24

25

26

27

28

29

30

31

32

33

34

35

36

37

38

39

40

41

42

43

44

45

46

47

48

49

50

51

52

53

54

55

56

57

58

59

60

61

62

63

64

65

\begin{tabular}{|c|c|c|}
\hline & IFS & FC \\
\hline Positive & 80 & 88 \\
\hline Negative & 340 & 332 \\
\hline
\end{tabular}


Click here to download high resolution image
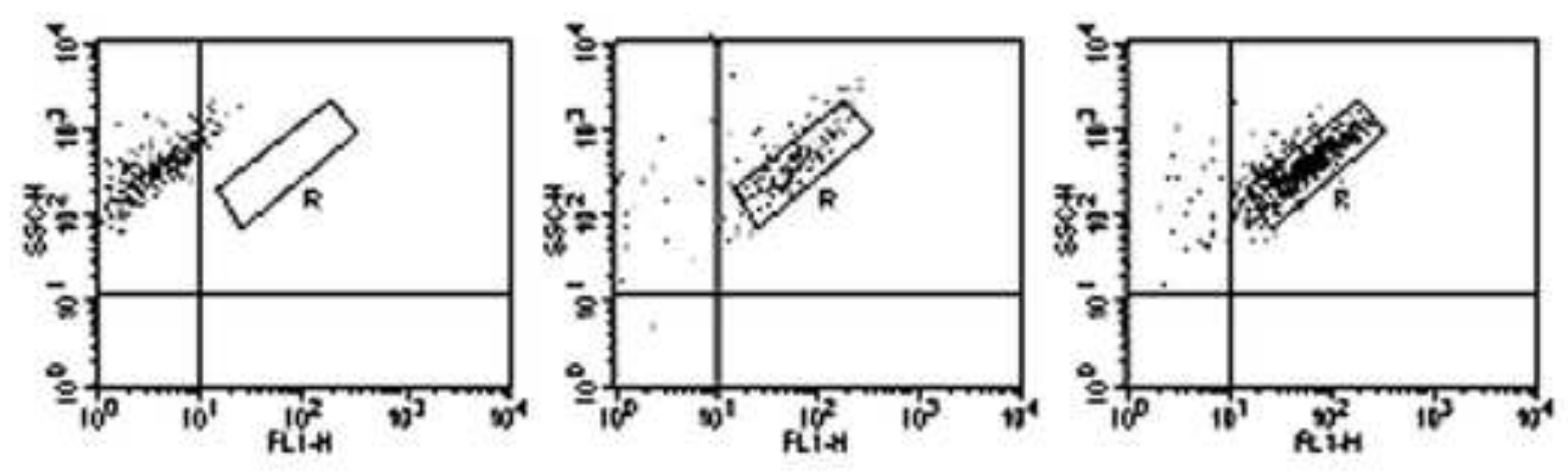
Click here to download high resolution image
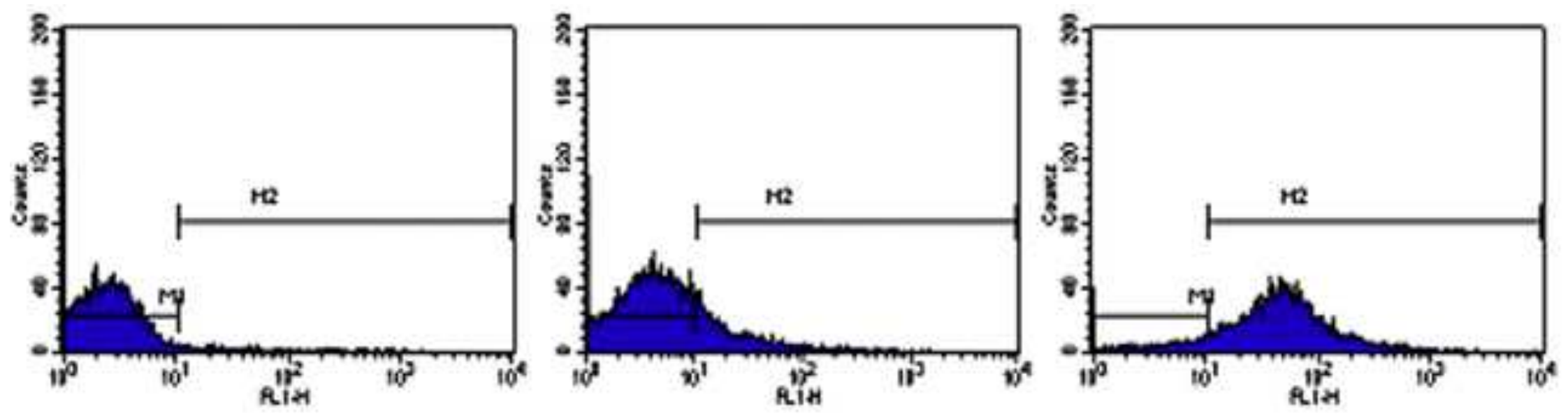
Click here to download high resolution image

\begin{tabular}{|cc|c|}
\hline & IFS & FC \\
\hline Positive & 80 & 88 \\
Negative & 340 & 332 \\
\hline
\end{tabular}

Positive

340

332 


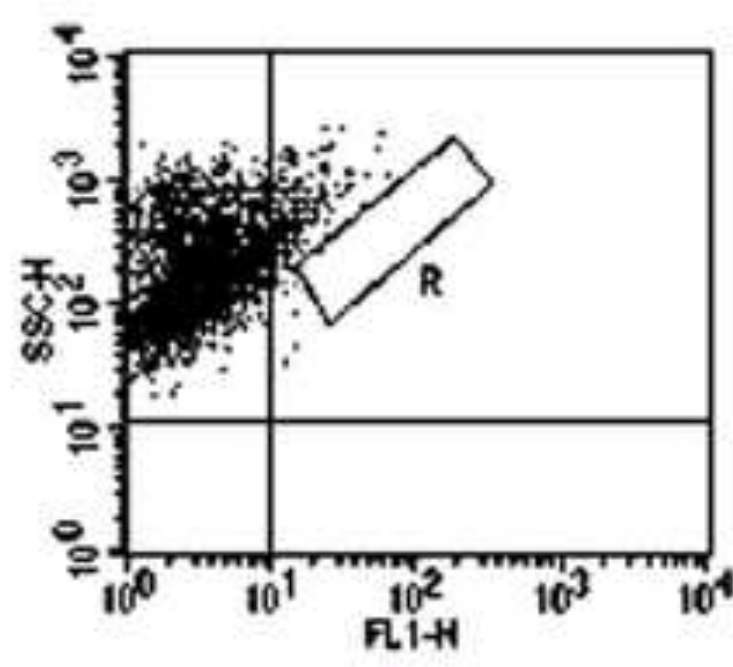

A

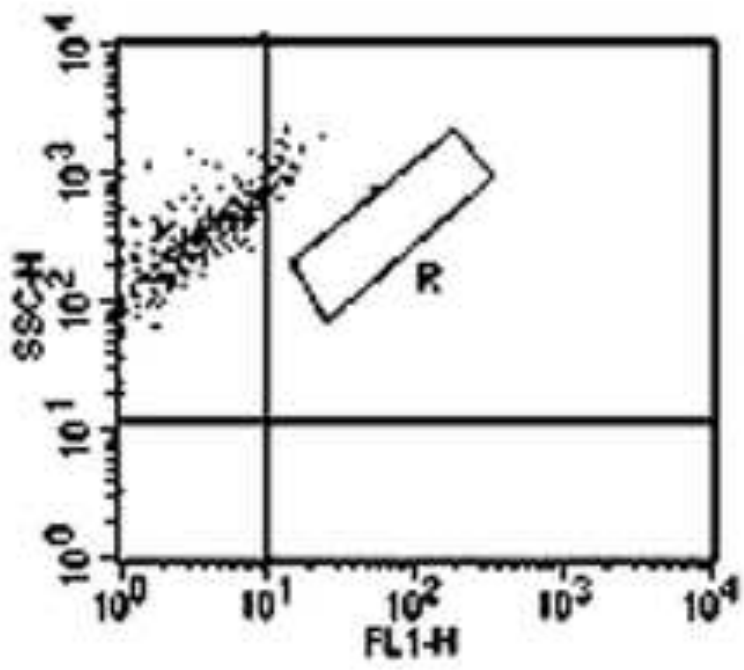

$\mathrm{C}$

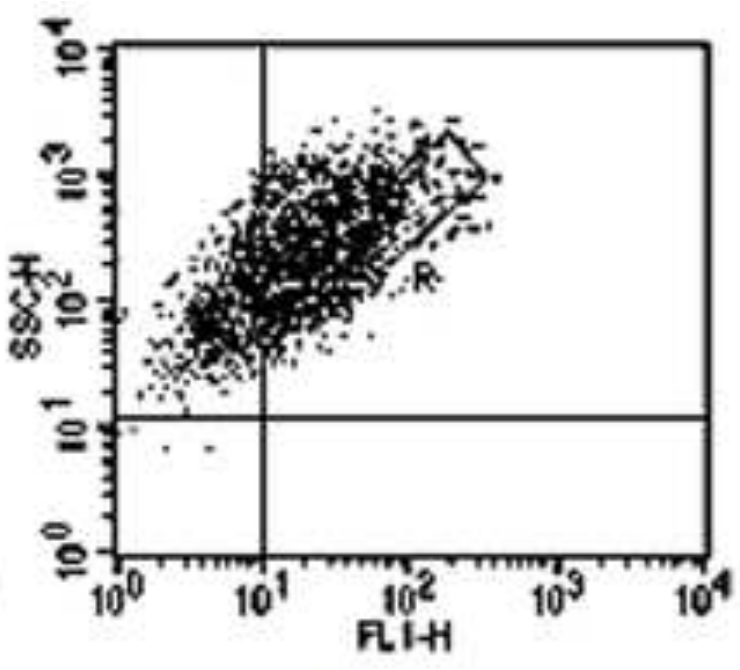

B

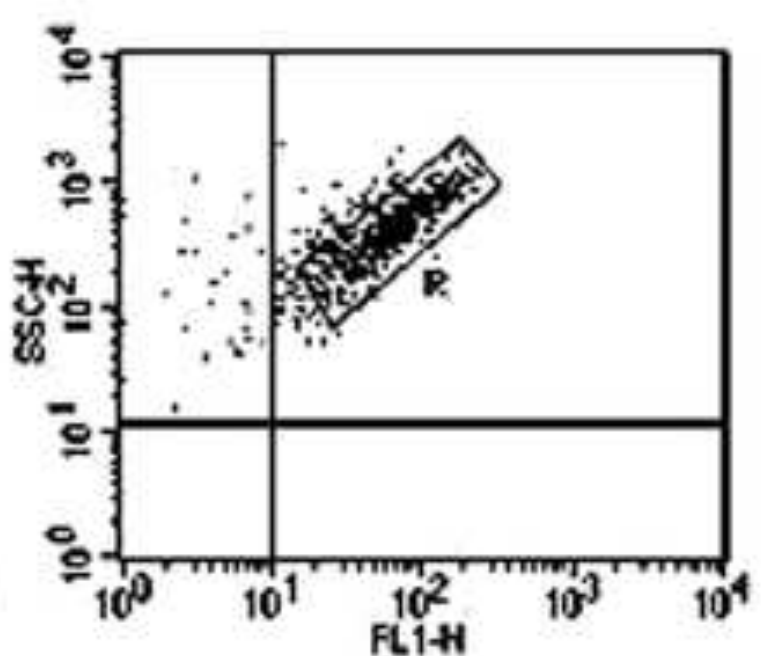

D 